\title{
ANALYSIS OF RECENT POLAR MOTION DATA IN VIEW OF EXCITATION FUNCTION AND POSSIBLE SEISMIC CAUSES
}

\author{
H. Lenhardt and E. Groten \\ Institute of Physical Geodesy \\ Petersenstraße 13 \\ 6100 Darmstadt \\ Germany
}

\begin{abstract}
Recent BIH-data from September 1979 - September 1986 have been deconvolved. The excitation function has been considered in detail and a spectral analysis indicates that there are atmospheric contributions; moreover, the September 19, 1985 Mexican Earthquake has been related to polar motion and a possible tidal triggering mechanism has been investigated.
\end{abstract}

\section{INTRODUCTION}

When we try to explain or interpret the polar motion results several quite different attitudes are possible: First of all, in case of BIH data we have to be aware of the fact that they are smoothed results. Stronger smoothing enhances the probability that the desired signal has been eliminated or reduced. This is important if we subtract directly the angular momentum effect of the atmosphere and/or the effect due to earthquakes and then interpret the residuals in view of other sources. A first candidate, as far as other sources of energy transfer are concerned, is the ocean but, contrary to seismic and atmospheric data, the present information on ocean mass changes with time, associated friction effects etc. is still too scarce.

On the other hand, we might be overcautious by assuming that we do not know exactly the energy transfer of seismic and atmospheric energy - also in view of uncertainties in separating smaller from larger earthquakes - and confine the investigation to crosscorrelation and spectral analysis by basically relating polar motion results with those of atmospheric angular momentum variations or scalar seismic moments. This is mainly the principle on which the present paper has been based because the seismic energy appears to be much to small for explaining variations in polar motion data; in that context, spectral analysis is mainly of interest for the investigation of atmospheric effects whereas the individual treatment of large earthquakes is of primary importance in case of seismic data. 
As far as tidal triggering of earthquakes is concerned we should take into account that in the superimposed lunar and solar tides a maximum occurs within about a month as a consequence of the different tidal frequencies. Besides diurnal or semidiurnal tidal variations this maximum should play a role if tidal triggering is really in existence.

Subsequently, we shall spotlight some aspects of the Chandler wobble excitation process in view of numerical techniques as well as of possible geophysical causes. For more details concerning the filter techniques we refer to a preceding paper (Lenhardt and Groten, 1986) as well as to related publications, e.g. (Gross and Chao, 1985) and (Smylie et al., 1970).

\section{DECONVOLUTION - A WAY TO OBTAIN THE EXCITATION SERIES}

Integration of the Chandler wobble equation of motion gives

$$
m(t)=m_{0} \cdot e^{i \sigma t}-i \sigma \int_{-\infty}^{t} \psi(\lambda) \cdot e^{i \sigma(t-\lambda)} d \lambda
$$

It can be seen that the second part on the right side is the convolution of the excitation function with the impulse response of the Earth. Consequently, the complex excitation function $\psi(t)$ may be obtained by a deconvolution, a numerical procedure which is most effectively performed in the frequency domain. Fourier transforming the Chandler wobble equation of motion gives immediately, by using the derivation theorem,

$$
M(\omega)=\Psi(\omega) \cdot G(\omega)+N(\omega)
$$

where a noise term $N(\omega)$ has been included. In principle, it is straightforward to obtain $\Psi(\omega)$ by choosing a filter transfer function $\mathrm{V}(\omega)$ being the inverse of the Earth's transfer function $G(\omega)=\sigma / \sigma-\omega$ ( $\sigma=$ complex Chandler frequency). However, because the transfer function $G(\omega)$ has a pronounced peak at the Chandler frequency (expressing the system resonance) its inverse will suppress the Chandler band by simultaneously amplifying the probably noise-dominated high frequency contributions. It is clear that this simple inverse filter has the undesirable property to act as a high-pass filter.

Obviously, a filter transfer function $V(\omega)$ has to be chosen which suppresses the noise amplification but succeeds as much as possible in producing a "white" spectrum for the Earth's transfer function. Both requirements (noise suppression and spectral whitening) are in conflict and a compromise may be found by introducing a Gaussian filter with a "free" parameter $k$ for the width of the Gaussian curve in the frequency domain (Deregowski, 1971). A very large value for $k$ determines a broad 
Gaussian curve which approximates complete spectral whitening whereas a narrow curve (small $\mathrm{k}$ ) attenuates the probably noise-dominated high frequencies but affects adversely the spectral whitening process. There are no "objective" criteria for the choice of $k$ and it will be necessary to compare the results obtained for different values of $k$.

\section{DATA PROCESSING}

The BIH data from September 1979 - September 1986 (Table 6 of the Annual Reports and Circular D) have been detrended and the annual wobble component has been removed by a least squares fit. For the Earth's transfer function the complex Chandler frequency has to be specified. Least-squares fitting yields 436 solar days. The quality factor $Q$ is of minor influence and it will be appropriate to take $Q=100$.

Figures 1 and 2 demonstrate the movement of the excitation pole as obtained by a simple inverse filter (left) and a strongly noise-stabilized Gaussian filter $(k=64)$. Please note the difference in scale.
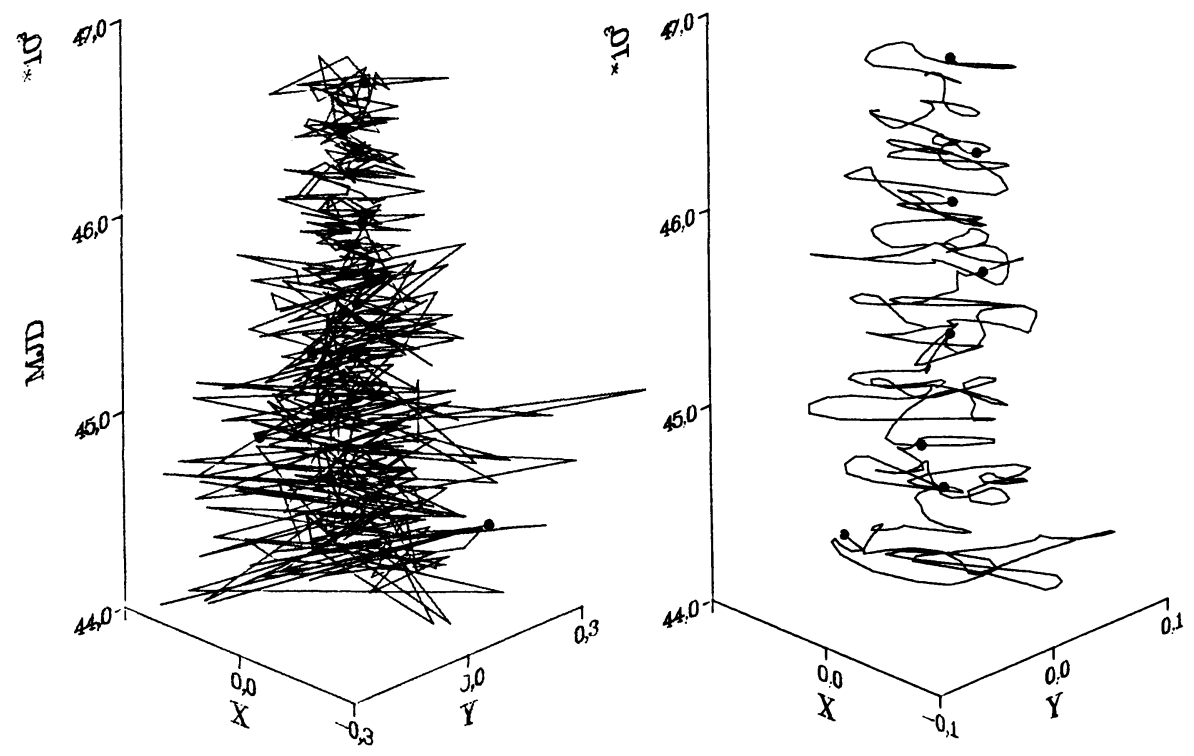

Figs. 1 and 2: Movement of the excitation pole (Sept 1979 - Sept 1986)

This time domain representation enables us to fix extraordinary events, for example the possible effect of a single large earthquake. A comparison of the $\mathrm{x}$ - and $\mathrm{y}$-component with the two extraordinarily large earthquakes which occured in 1985 (Valparaiso, March 3 and Michoacan, Sept 19) gives, however, no indication for a perceptible interaction. 
(Gross, 1986) as well as (Souriau and Cazenave, 1985) investigated the earthquake excitation theory but concluded finally that the order of magnitude of the excitation is far too small to have a significant influence. As an example, we calculate the effect of the Michoacan event of Sept 19, 1985 by using the formulae given by (Wahr, 1983), (Dahlen, 1973) and (Gross, 1986). The moment tensor components (CMTsolution) were published by (Ekström and Dziewonski, 1986) and a theoretical shift of the excitation pole of 0.000096 arcsec is obtained which is certainly not observable.

The frequency domain representation of the excitation process shows the frequencies which are delivering most of the energy. As the resolution decreases rapidly for the lower harmonics by using only seven years of data we focus on frequencies with corresponding periods between 20 and 200 days. The spectral whitening process described above moves along with a significant loss of coherent signal energy (Bowen, 1986) and the spectrum becomes flat. For a graphic representation it will be favourable to use spectral estimators which produce pronounced peaks. Particularly the maximum entropy spectral estimator is known to fulfill this requirement and Fig. 3 shows the spectral density of the excitation series ( $x$-component) in the case where a weak noise stabilization has been set up.

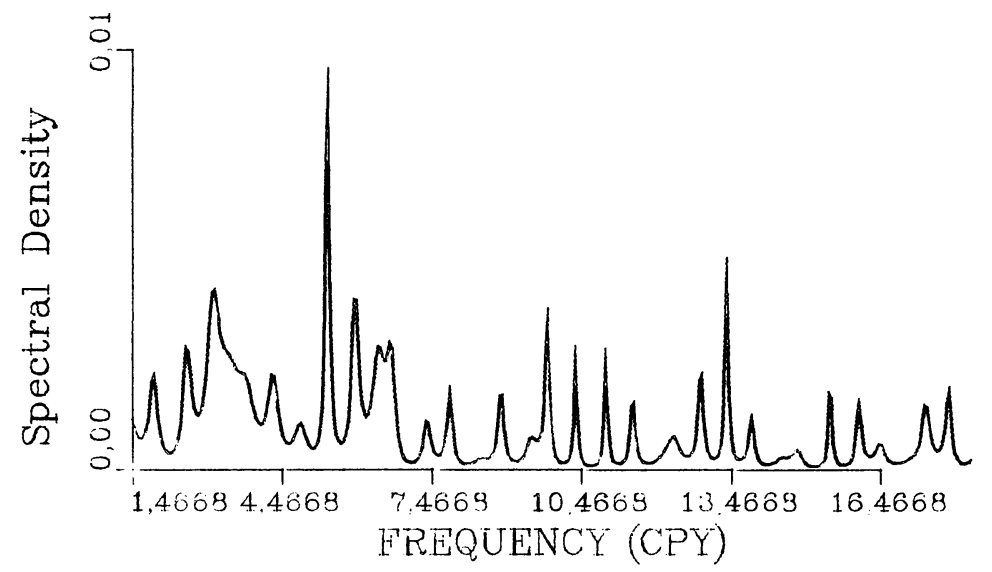

Fig. 3: Maximum entropy spectrum of the excitation series ( $x$-comp.) ( A 147-point operator has been applied)

Between the two dominating frequencies with corresponding periods of 67.8 days and 27.3 days several spectral peaks can be seen (with corresponding periods of $61.8,57.2,55.1,49.7,46.7,41.2,37.3,35.3$, $33.4,31.7,29.7$ and 28.4 solar days). One may speculate that these peaks might be due to atmospheric effects which have been discussed for lod (length of day) fluctuations, see e.g. (Eubanks et al., 1985). We may not presume that the spectral representation of angular momentum of the atmosphere is completely constant. 
Besides computational effects this could explain differences between our present results and those given in (Lenhardt and Groten, 1986).

\section{TIDAL TRIGGERING AND THE MICHOACAN EVENT}

(Ekman, 1985) concluded that a major earthquake can be triggered by the combined semi-diurnal and diurnal earth tide in such a way that the earthquake is more likely to occur when the tidal curvature is increasing than when it is decreasing, provided the Gaussian tidal curvature $\mathrm{K}$ exceeds about $1.5 \cdot 10^{-34} \mathrm{~mm}^{-2}$. As already stated above, the Michoacan event of Sept 19, 1985 with a scalar seismic moment of $1.03 \cdot 10^{28}$ dyne$\mathrm{cm}$ (Ekström and Dziewonski, 1986) was one of the largest earthquakes during the last decade. The Gaussian tidal curvature $\mathrm{K}$ referred to this event according to the formula given by (Ekman, 1985) is shown in Fig. 4 in dependence of Universal Time (Sept 19, 1985).

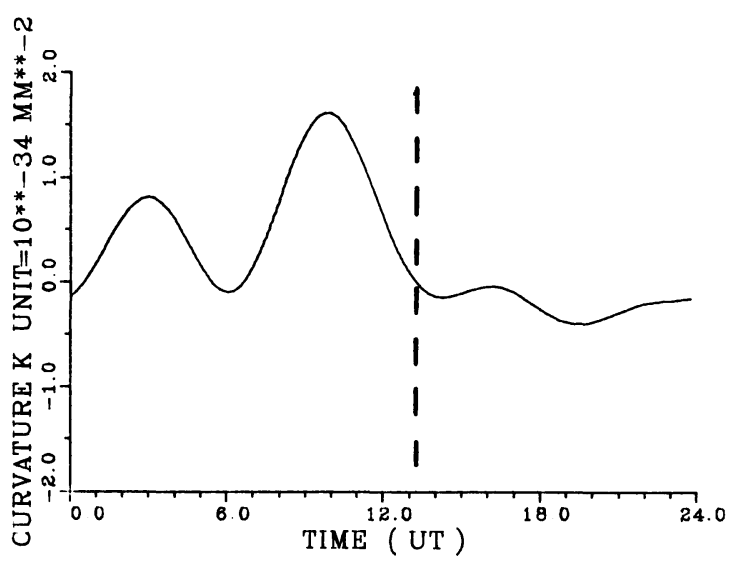

Fig. 4: Gaussian tidal curvature (date and site of the Michoacan event)

It can be seen that $K$ reaches its maximum value of $1.61 \cdot 10^{-34} \mathrm{~mm}^{-2}$ at about 9:45 UT whereas the Michoacan event occured at 13:17:49.1 UT, nearly exactly when the Gaussian tidal curvature becomes negative.

Consequently, the statistically founded rule put forward by (Ekman, 1985) is not backed in this case but one example only can certainly not give much additional information.

\section{CONCLUSIONS}

A comparison of the excitation function which has been obtained by deconvolving relatively few but "accurate" BIH data with different types of seismic information gives no indication for an interaction of significant impact. However, it must be emphasized that even the 
largest earthquake which occured in the time interval under investigation is assumed to have at least one magnitude less than, for example, the Alaskan earthquake of 1964.

The spectral peaks in the frequency domain representation of the excitation function with corresponding periods between 30 days and 70 days imply that they are caused by atmospheric processes. Attenuation due to the Earth's transfer function would make their influence on the Chandler motion, however, negligibly small. Other sources such as oceanic effects may contribute to the Chandler motion and there is a need of substantially better polar motion data and other contributing data.

\section{REFERENCES}

Bowen A.N. (1986) 'A Comparison of Statistical and Deterministic Wiener Deconvolution of Deep-Tow Seismic Data'. Geophysical Prospecting 34, pp. 366-382.

Dahlen F.A. (1973) 'A correction to the excitation of the Chandler Wobble by earthquakes'. Geophys. J,R. astr. Soc. 32, pp. 203-217.

Deregowski S.M. (1971) 'Optimum Digital Filtering and Inverse Filtering in the Frequency Domain'. Geophysical Prospecting 19,pp.729-768.

Ekman M. (1985) Gaussian and Mean Curvatures of Earth Tides and Postglacial Land Uplift, and their Effects on Earthquakes, Doctoral dissertation. University of Uppsala, Dep. of Geodesy.

Ekström G. and Dziewonski A.M. (1986) 'A, Very Broad Band Analysis of the Michoacan, Mexico, Earthquake of September 19, 1985'. Geophysical Research Letters, Vol. 13, No. 6, pp. 605-608.

Eubanks T.M., Steppe J.A., Dickey J.0. and Callahan P.S. (1985) 'A Spectral Analysis of the Earth's Angular Momentum Budget'. Journal of Geophysical Research, Vol. 90, No. B7, pp. 5385-5404.

Gross R.S. (1986) 'The influence of earthquakes on the Chandler wobble during 1977-1983'. Geophys. J.R. astr. Soc. 85, pp. 161-177.

Gross R.S. and Chao B.F. (1985) 'Excitation Study of the LAGEOS-Derived Chandler Wobble'. Journal of Geophysical Research, Vol. 90, No. B11, pp. 9369-9380.

Lenhardt H. and Groten E. (1986) 'Polar Motion Analysis' . Paper presented at the International Symposium "Figure and Dynamics of the Earth, Moon and Planets", Prague 1986.

Smylie D.E.,Clarke G.K.C and Mansinha L. (1970) 'Deconvolution of the Pole path'. Earthquake Displacement Fields and the Rotation of the Earth. Edited by L. Mansinha, D.E. Smylie and A.E. Beck, D. Reidel Publishing Company, Dordrecht-Holland.

Souriau A. and Cazenave A. (1985) 'Reevaluation of the Chandler wobble seismic excitation from recent data'. Earth and Planetary Letters, 75 , pp. 410-416.

Wahr J.M. (1983) 'The effects of the atmosphere and oceans on the Earth's wobble - I. Theory'. Geophys. J.R. astr. Soc.. 70, pp. 349-372.

\section{DISCUSSION}

Débarbat: You have made a global study of this problem. The local conditions could vary. As an example, during the last major earthquake in Santiago de Chile, the latitude changed by 1.5 meters. 